\title{
Iris Melanoma in a Patient with Retinitis Pigmentosa
}

\author{
Hiroshi Goto Naoyuki Yamakawa \\ Department of Ophthalmology, Tokyo Medical University, Tokyo, Japan
}

\section{Established Facts}

- Iris melanoma is a relatively rare intraocular malignant tumor, especially in Asia.

- Retinitis pigmentosa (RP) is an inherited disorder that causes loss of vision and night blindness.

- A previous case report described choroidal melanoma in a patient with RP.

\section{Novel Insights}

- This is the first case report describing an iris melanoma that developed in a patient with retinitis pigmentosa.

\section{Keywords}

Iris melanoma $\cdot$ Retinitis pigmentosa $\cdot$ Histopathology

\section{Abstract}

This report describes the histopathological findings of an iris melanoma that developed in a patient with retinitis pigmentosa (RP). A 66-year-old man was referred to our hospital with a diagnosis of iris tumor. He had a medical history of RP for over 25 years. His best corrected visual acuity at presentation was 20/20 (OD) and 20/200 (OS). Slit lamp examination revealed an iris melanocytoma in his right eye and a brown iris mass involving the pupil in his left eye. Ocular fundus examination of his right eye showed diffuse chorioretinal atrophy with attenuated retinal vessels and scattered pigment. A diagnosis of iris melanoma was made and enucleation of his left eyeball was performed. Histopathological examination of the eyeball showed an iris tumor with proliferation of spindle cells positive for Melan-A, HMB-45, and S-100 protein by immunohistochemistry. Diffuse destruction of retinal ar- chitecture was observed, with loss of outer segment and replacement by glial cells. Accumulation of melanin pigment around retinal vessels was found in peripheral retina. These histopathological findings were compatible with advanced stage of RP. This case demonstrates that iris melanoma can occur in eye with RP.

(C) 2021 The Author(s) Published by S. Karger AG, Basel

\section{Introduction}

Iris melanoma constitutes a minority of uveal melanoma even in North America and Europe [1-4] and is extremely rare in Asia. Histopathologically, the most common type of iris melanoma is composed of spindle-shaped cells, followed by the mixed-cell type consisting of spindle and epithelioid cells. In general, metastasis of iris melanoma is quite rare $[5,6]$, although extraocular extension and high intraocular pressure are considered to be risk factors of metastasis [2]. Retinitis pigmentosa (RP) is charac-

(C) 2021 The Author(s)

Published by S. Karger AG, Basel

This is an Open Access article licensed under the Creative Commons Attribution-NonCommercial-4.0 International License (CC BY-NC) (http://www.karger.com/Services/OpenAccessLicense), applicable to the online version of the article only. Usage and distribution for commercial purposes requires written permission.
Correspondence to:

Hiroshi Goto, goto1115@ tokyo-med.ac.jp 
Fig. 1. Slit lamp examination showing a well-defined melanocytic lesion on the depigmented iris in the right eye (a), and a brown irregularly shaped iris mass covering the pupil in the left eye (b).
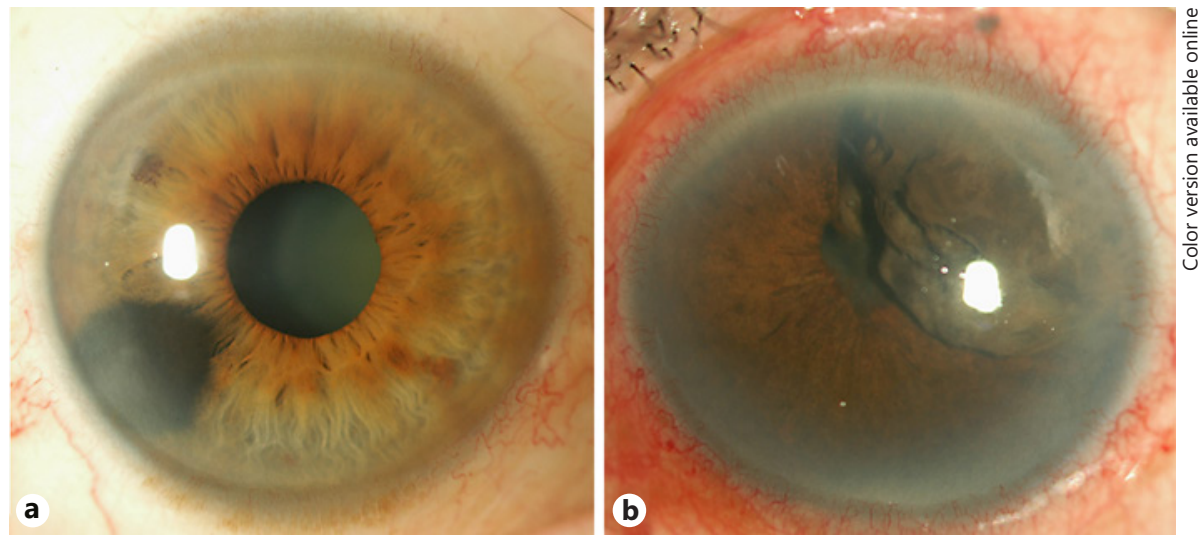
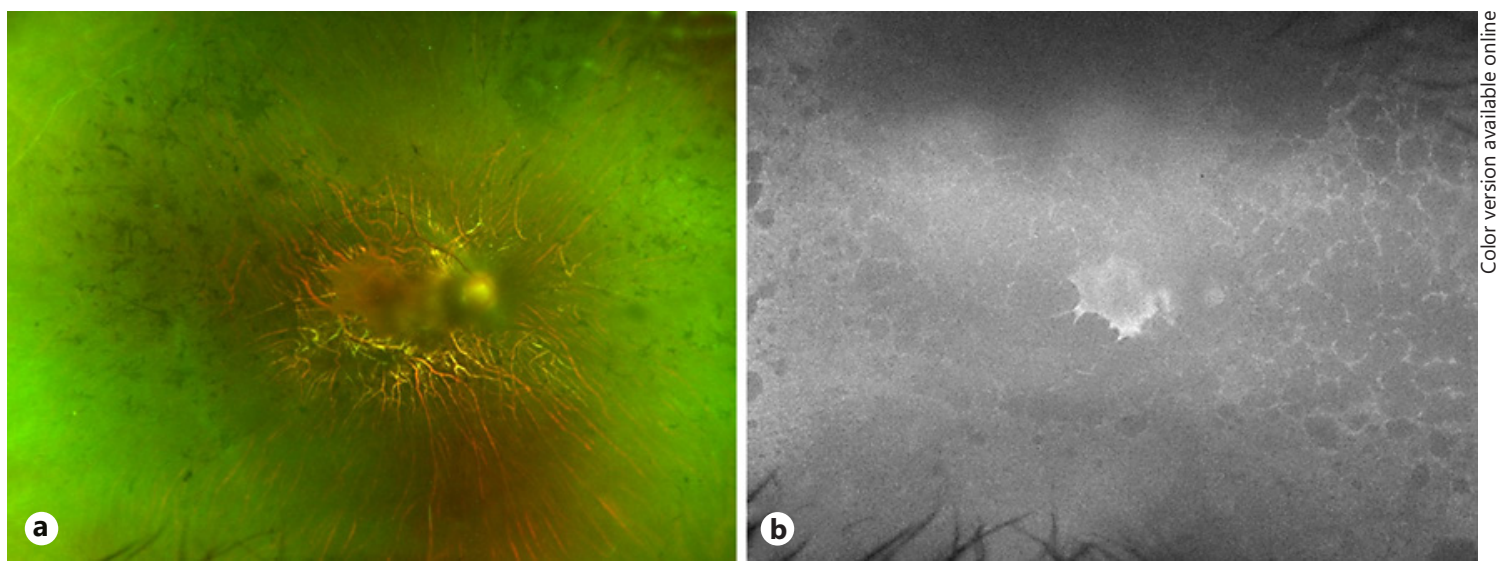

Fig. 2. Ocular fundus photograph and FAF image of the right eye. a Note diffuse chorioretinal atrophy with attenuated retinal vessels and scattered bone-corpuscular pigment at the periphery. b FAF image shows diffuse patchy hypofluorescence except in the macular area. FAF, fundus autofluorescence.

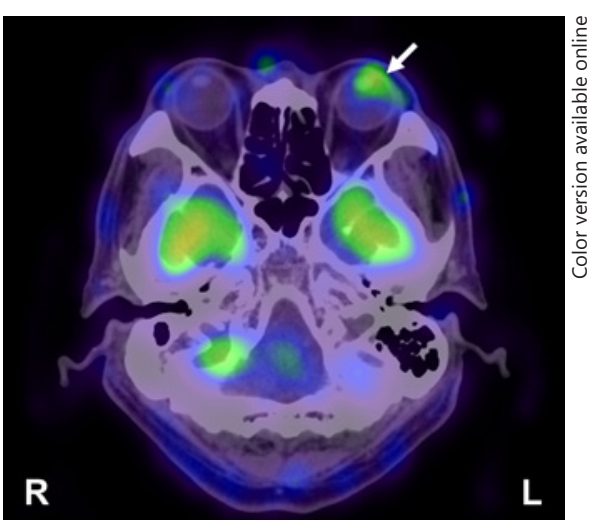

Fig. 3. SPECT using ${ }^{123} \mathrm{I}$-IMP is positive in the left eye (arrow), but negative in the right eye. SPECT, single-photon emission computed tomography; ${ }^{123} \mathrm{I}-\mathrm{IMP}, \mathrm{N}$-isopropyl- $p$-[ $\left.{ }^{123} \mathrm{I}\right]$ iodoamphetamine. terized by diffuse, usually bilateral retinal dystrophy causing night blindness and visual field loss. Central vision tends to be maintained until the late stage. Although cystoid macular edema and posterior subcapsular cataract are common in the longstanding clinical course of RP, severe complications such as uncontrolled glaucoma with painful eye requiring eyeball enucleation are rarely encountered. To the best of our knowledge, no case report of iris melanoma developed in a patient with RP has been published. We report the clinical and histopathological findings of iris melanoma developed in a patient with advanced RP.

\section{Case Report}

A 66-year-old Japanese man was referred to the Department of Ophthalmology, Tokyo Medical University Hospital, in December 2017 with a clinical diagnosis of iris tumor in the left eye. He had a medical history of RP for over 25 years without hearing loss or 

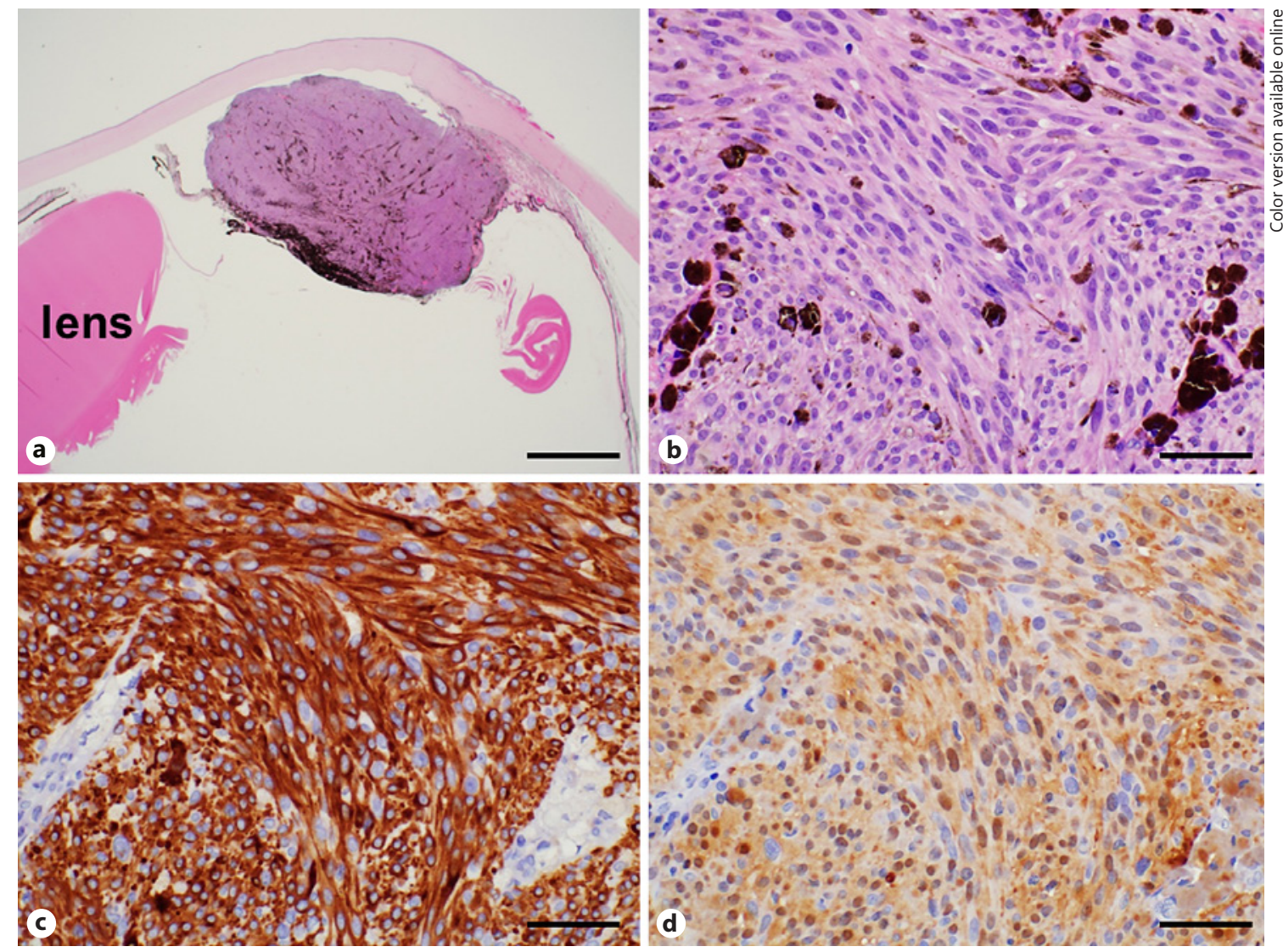

Fig. 4. a Histopathology of the anterior segment of the enucleated eyeball. Note the pigmented tumor on the iris with invasion into trabecular meshwork (hematoxylin-eosin staining, bar: $1.5 \mathrm{~mm}$ ). b Tumor is composed of spindle cells with high nucleus to cytoplasm ratio and prominent nucleoli (hematoxylin-eosin staining, bar: 50 $\mu \mathrm{m})$. Immunohistochemical staining of the tumor cells are diffusely positive for Melan-A (c), S-100 protein (d) (bar: $50 \mu \mathrm{m}$ ).

systemic disease. His family history was unremarkable. He was not followed periodically for RP. His best corrected visual acuity at presentation was 20/20 (OD) and 20/200 (OS). Intraocular pressure was $14 \mathrm{~mm} \mathrm{Hg}(\mathrm{OD})$ and $19 \mathrm{~mm} \mathrm{Hg}(\mathrm{OS})$. Slit lamp examination revealed a well-defined melanocytic lesion on the depigmented iris, presumably melanocytoma, in his right eye (shown in Fig. 1a), and a brown irregularly shaped iris mass extending from 12 to 4 o'clock in his left eye (shown in Fig. 1b). Ocular fundus examination of his right eye showed diffuse chorioretinal atrophy with attenuated retinal artery and vein and scattered bone-corpuscular pigment at the periphery (shown in Fig. 2a). Fundus autofluorescence imaging depicted diffuse patchy hypofluorescence except in the macular area (shown in Fig. 2b). Detailed ocular fundus finding in his left eye could not be visualized due to the iris mass hanging over the pupil and posterior synechiae. Goldmann perimetry of the right eye showed central constriction of visual fields to less than $10^{\circ}$ (V/4 index). Ultrasonography and magnetic resonance imaging showed no significant changes in the left eye except for the iris mass. Single-photon emission computed tomography using $\mathrm{N}$-isopropyl- $p-\left[{ }^{123} \mathrm{I}\right]$ iodoamphetamine, which is useful for the diagnosis of uveal melanoma [7] was positive in the left eye, but negative in the right eye (shown in Fig. 3). Four weeks later, the size of the iris mass in his left eye apparently enlarged. Visual acuity decreased to hand motion and intraocular pressure increased to $27 \mathrm{~mm} \mathrm{Hg}$. A diagnosis of iris melanoma was made based on the clinical manifestation and clinical course. Subsequently, enucleation of the left eyeball was performed with the patient's informed consent.

Histopathology of the anterior segment is given in Figure 4. An oval tumor containing melanin pigment was observed on the iris. Slight invasion of the tumor into the trabecular meshwork was observed, but the ciliary body was not involved. The iris tumor contained proliferated spindle cells with high nucleus to cytoplasm ratio and prominent nucleoli. Epithelioid cells were occasionally observed. In immunohistochemical study, the tumor cells were diffusely positive for Melan-A and S-100 protein, and partially positive for HMB-45. The proportion of Ki-67-positive cells was approximately $5 \%$ under high power magnification. These histopathological and immunohistochemical findings were compatible with a diagnosis of iris melanoma.

Histopathology of the retina and choroid is shown in Figure 5. The retinal architecture was completely destroyed in all layers 

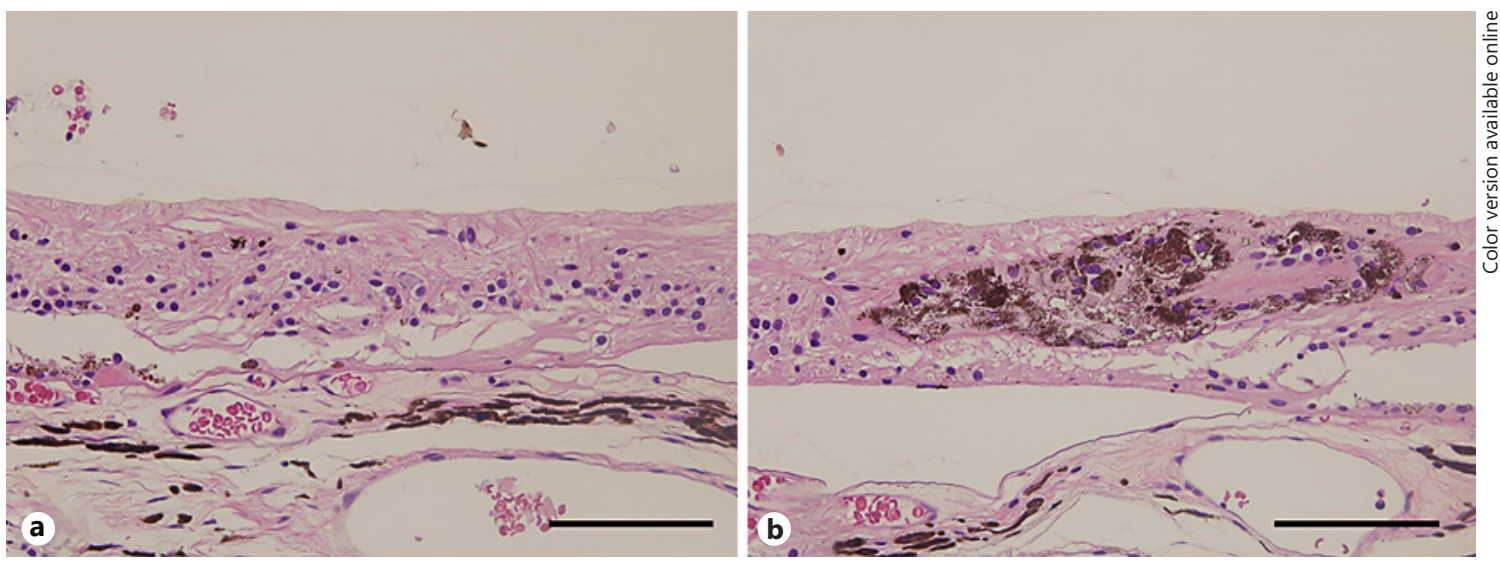

Fig. 5. Histopathology of the retina and choroid. Retinal architecture is destroyed in all layers and replaced by glial cells. RPE is atrophic at the posterior pole (a) and is absent at the periphery (b). Accumulation of melanin pigment around obstructed retinal vessels is observed at the peripheral retina. Choriocapillaris is totally absent and dilated choroidal vessels are observed (hematoxylin-eosin staining, bar: $100 \mu \mathrm{m}$ ). RPE, retinal pigment epithelium.

spanning from the posterior pole to periphery, and was replaced by glial cells. Most of the retinal pigment epithelium was atrophic and hypopigmented at the posterior pole and was absent at the periphery. Accumulation of melanin pigment around obstructed retinal vessels was observed at the peripheral retina. Choriocapillaris was totally absent while dilated choroidal vessels were found. These retinal and choroidal histopathological findings were compatible with advanced stage of RP.

Monosomy 3 was not detected in the iris tumor by fluorescence in situ hybridization. The patient has been followed at a referral hospital for 29 months after eyeball enucleation and extraocular metastasis has not been detected. The melanocytic iris tumor in his right eye has not changed in size or color.

\section{Discussion}

Shields et al. [3] analyzed 8,033 cases of uveal melanoma and reported that iris melanoma represented only $4 \%$, compared with ciliary body (6\%) and choroid (90\%). Among 187 cases of uveal melanoma diagnosed either clinically or histopathologically in our hospital, 178 cases (95\%) were choroidal melanoma, 6 cases (3\%) were ciliary body melanoma, and only 3 cases (2\%), including the present case were iris melanoma. Iris melanoma tends to occur in the inferior portion of the iris [2]. Interestingly, all 3 Japanese patients we encountered had iris melanoma located in the superior aspect of the iris. In addition, all 3 patients required enucleation of the eyeball due to aggressive growth within a short period, although MIB-1 proliferation index (proportion of Ki-67-positive tumor cells) were less than $5 \%$ in all cases. These findings may suggest differences in pathogenesis of iris melanoma among races.

A case report showed that choroidal melanoma caused pseudo-RP by retinal invasion and dispersion of melanoma cells on the retina [8]. The present case, however, showed typical ocular fundus appearance compatible with a diagnosis of advanced RP. In the literature, 2 cases of uveal melanoma that developed in patient with RP have been reported $[9,10]$. The case reported by Gündüz et al. [10] was a choroidal melanoma associated with Usher's syndrome, which is characterized by RP and hearing loss. The case of Lommatzsch et al. [9] was also a choroidal melanoma associated with pseudo-RP. On the other hand, the present case was an iris melanoma that developed in a patient with sporadic RP, and both diagnoses were histopathologically proven.

The association between uveal melanoma and RP is obscure. The fact that such cases were rarely reported may suggest no particular association in pathogenesis between these 2 clinical entities. Incidentally, our patient also had a melanocytic iris tumor, presumably melanocytoma, in his right eye. The origin of the iris melanoma in his left eye is unknown because periodical ophthalmic examination had not been performed despite the long medical history of RP. Shields et al. [11] reported that iris nevus growth into melanoma occurred in less than $1,3,4,8$, and $11 \%$ of the cases at $1,5,10,15$, and 20 years, respectively. Therefore, careful follow-up of our patient is required not only for RP but also for the melanocytic lesion in the iris in his remaining eye. 


\section{Statement of Ethics}

Written informed consent was obtained from the patient for publication of this case and any accompanying images. Ethical approval for this case report was exempted by the institutional review board at our institution.

\section{Conflict of Interest Statement}

The authors have no conflicts of interest to declare.

\section{Funding Sources}

No funding was received.

\section{Author Contributions}

Hiroshi Goto: conceptualization, data interpretation, and drafting of the paper. Naoyuki Yamakawa: data preparation and interpretation and Revision of the draft paper.

\section{References}

1 Shields CL, Furuta M, Thangappan A, Nagori S, Mashayekhi A, Lally DR, et al. Metastasis of uveal melanoma millimeter-by-millimeter in 8,033 consecutive eyes. Arch Ophthalmol. 2009;127(8):989-98.

2 Shields CL, Kaliki S, Shah SU, Luo W, Furuta M, Shields JA. Iris melanoma: features and prognosis in 317 children and adults. J AAPOS. 2012;16(1): 10 .

3 Shields CL, Kaliki S, Furuta M, Mashayekhi A, Shields JA. Clinical spectrum and prognosis of uveal melanoma based on age at presentation in 8,033 cases. Retina. 2012;32(7):136372.
4 Shields CL, Kaliki S, Cohen MN, Shields PW, Furuta M, Shields JA. Prognosis of uveal melanoma based on race in 8,100 patients: the 2015 doyne lecture. Eye. 2015;29(8):1027-35.

5 Arensten JJ, Green WW. Melanoma of the iris: report of 72 cases treated surgically. Ophthalmic Surgery. 1975;6:23-37.

6 Sunba MS, Rahi AH, Morgan G. Tumors of the anterior uvea. Metastasizing malignant melanoma of the iris. Arch Ophthalmol. 1980; 98:82-5.

7 Goto H, Usui M, Ishii I. Efficacy of (123) Nisopropyl-p-[(123)I]-iodoamphetamine single photon emission computed tomography for the diagnosis of uveal malignant melanoma. Am J Ophthalmol. 2001;132:937-39.
8 Eagle RC Jr, Shields JA. Pseudoretinitis pigmentosa secondary to preretinal malignant melanoma cells. Retina. 1982;2(1):51-5.

9 Lommatzsch PK, Jensen OA, JU Prause, Bauke G. Pseudoneuritis pigmentosa in malignant melanoma of the choroid. Klin Monbl Augenheilkd. 1988;193(1):69-74.

10 Gündüz K, Shields JA, Shields CL, Zhao DY. Choroidal melanoma in a patient with retinitis pigmentosa and usher's syndrome. $\mathrm{Br} \mathrm{J}$ Ophthalmol. 1998;82(7):847-8.

11 Shields CL, Kaliki S, Hutchinson A, Nickerson S, Patel J, Kancherla S, et al. Iris nevus growth into melanoma: analysis of 1,611 consecutive eyes: the ABCDEF guide. Ophthalmology. 2013;120(4):766-72. 